Aims 34,000 Children and Young People (CYP) in England have a diagnosis of epilepsy and receive anti-epileptic drugs. NICE have described high-priority areas for quality improvement; The Epilepsy in CYP, Quality Standard (QS27).

Our local service aims to provide an excellent multidisciplinary service to serve its patients and their families in a holistic manner. It is delivered by consultants, a specialist nurse, rotating junior doctors and the nursing team.

Methods To evaluate our current local service standard against national guidance and identify areas of possible improvement, we developed a Caregiver and Patient Reported Survey (CPRS). It covered both quality standards and expectations from patients and their parents and caregivers. Anonymous CPRS were disseminated in the waiting areas of our mutli-site outpatient paediatric epilepsy clinics over a 3 month period $(n=44)$. Observational analyses were completed on all the returned CPRS and the results were mapped against national guidance.

Results Observational analyses revealed $70.5 \%$ of CPRS were completed by caregiver/parent and $22.7 \%$ by CYP $(6.8 \%$ no return), $84.1 \%$ stated that they were satisfied-very satisfied with our services, $90.9 \%$ were happy-very happy with their clinical experience, $82.7 \%$ were happy- very happy with the support they received outside of the clinic environment (34.0\% no return) and $77.8 \%$ were happy- very happy with the support received whilst inpatients (38.6\% no return).

Our patients had experienced problems in the educational, social and home lives due to epilepsy; $31.8 \%$ at school, $18.2 \%$ attending out of school clubs and $31.8 \%$ socialising. $18.2 \%$ of patients experienced problems with other areas including; side effects of medication, communication with services and behavioural issues. 90.2\% were content with the information they received following diagnosis and $95.2 \%$ between appointments. $86 \%$ were happy to very happy with the verbal information and $81.4 \%$ with written information.

$14 \%$ identified areas of change including; information on the effects of epilepsy on learning and education, more streamlined information and flexibility around access to services.

Conclusion Communication and information delivery were identified as key areas of possible improvement. We are now developing an electronic, locally tailored Paediatric Epilepsy Information Pack to be disseminated to patients and their families.

\section{G326(P) IMPACT OF COMPLEX REHABILITATION USING ROBOTIC KINESIOTHERAPY AND BOTULINUM THERAPY ON THE LEVEL OF SOCIAL ADAPTATION OF CHILDREN WITH CEREBRAL PALSY}

${ }^{1} \mathrm{M}$ Balgayeva, ${ }^{2} \mathrm{Sh}$ Bulekbayeva, ${ }^{2} \mathrm{Zh}$ Daribayev, ${ }^{2} \mathrm{G}$ Zhylkybaev, ${ }^{2} \mathrm{M}$ Kenzhebekova ${ }^{1}$ Department of Neurology, ISC Astana Medical University, Astana, Kazakhstan; ${ }^{2}$ The National Children's Rehabilitation Centre, The Corporate Fund University Medical Centre, Astana, Kazakhstan

\subsection{6/archdischild-2018-repch.316}

Aims To assess the impact of complex rehabilitation using robotic kinesiotherapy, botulinum therapy in children with spastic diplegia on their social adaptation.

Methods The study involved 142 children with a clinical diagnosis: Cerebral palsy, spastic diplegia. Of these, 82 children were included in the main group, where robotic kinesiotherapy with the help of the Lokomat rehabilitation complex and intramuscular injection of botulinum toxin type A were performed as part of complex rehabilitation. The control group consisted of 60 children, who underwent rehabilitative treatment with traditional methods. According to the Gross Motor Functions Classification System (GMFCS) children of both groups are divided into 2 subgroups: II and III levels. To evaluate the effectiveness of the rehabilitation therapy complex the following tools were used: GMFCS, Modified Barthel Scale. Statistical analysis of the results of this study was carried out using IBM SPSS Statistics Professional 21.0.

Results There were no significant differences in age characteristics and gender between the study groups. The groups studied were also comparable in terms of motor deficit $(p=0.324)$. Identification of violations of self-service was carried out according to the following criteria: complete dependence, moderate dependence, easy dependence. It was revealed that children of both groups in terms of self-service and social adaptation are lagging behind the age norm. At the beginning of the therapy, there was no significant difference between the compared groups.

Improvement of self-care with the transition to an easier group was observed in 14 patients in the main group $(p<0.05)$, which significantly exceeded the results in the control group, where improvement was noted in 3 patients. At the end of the rehabilitation course, $71.9 \%$ of the patients in the main group had moderate dependence, $19.5 \%$ - easy dependence, complete dependence was maintained in $8.5 \%$ of patients. In the control group, $55 \%$ of patients had moderate dependence, complete dependence $21.6 \%$ and easy dependence $-23.3 \%$.

Conclusion Thus, the results of our study indicate a higher effectiveness of complex rehabilitation with the use of robotic kinesiotherapy and botulinum therapy in comparison with the methods of traditional therapy in patients with spastic diplegia.

\section{G327(P) CHARACTERISTICS AND OUTCOME OF HEADACHES IN CHILDREN FROM A TERTIARY CARE HEADACHE CLINIC}

A Michael, K Vraka, A Ponnampalam, W Whitehouse. Paediatrics, Nottingham University Hospital, Nottingham, UK

\subsection{6/archdischild-2018-rcpch.317}

Aim Headache is a common cause for referral to paediatric outpatients. Whilst there is clear guidance on recognition of patients intracranial causes of headache, the management and outcome of idiopathic headaches is variable.

Method We reviewed the characteristic of 28 patients from a tertiary headache clinic between 2013 and 2015. Demographics, diagnosis, indication for MRI, management and outcome of the patients was reviewed.

Result There was a female predominance of $61 \%$ with mean age of 12.8 years at initial appointment. $53 \%$ of patients were felt to have a chronic headache at presentation, $46 \%$ of which improved with treatment. The most frequent diagnosis was migraine without aura (57\%) and migraine with aura (25\%). Three patients were diagnosed with medication overuse headache, two with cluster headache, two with chronic paroxysmal hemicranias and one patient with each tension headache and idiopathic intracranial hypertension.

The majority of patients underwent MRI scan (89\%) of which $72 \%$ were reported as normal, $20 \%$ had small white matter changes that are often seen in patients with migraine and $8 \%$ had no change in previously known abnormalities. 
The main indication for MRI was headache waking at night $(16 \%)$ and an atypical headache (16\%). 32\% of patients had a previous MRI either for a different indication or done by the referring hospital prior to headache clinic.

Outcomes were positive with $71 \%$ of patients symptoms improving or completely resolving. $28 \%$ of patients had no improvement in symptoms. The most common treatments were Sumatriptan (43\% of patients) and Ibuprofen (26\% of patients), $21 \%$ of patients were medication free at the last appointment.

Conclusion Management of headache in children in our specialist tertiary clinic shows largely positive outcomes in improvement of symptoms. MRI was requested for majority of patients with no serious pathology identified in any patients. We suggest that while MRI is a useful tool, careful assessment and management of the symptoms may be able to avoid unnecessary investigations in majority some patients and improve their symptoms.

\section{G328(P) RISK OF TRANSVERSE MYELITIS FOLLOWING ZIKA VIRUS INFECTION}

${ }^{1} \mathrm{G}$ Oligbu, ${ }^{2} \mathrm{M}$ Fallaha, ${ }^{2} \mathrm{~L}$ Pay, ${ }^{3} \mathrm{~L}$ Ahmed, ${ }^{4} \mathrm{~A}$ Adepoju. ${ }^{7}$ Paediatric Infectious Diseases Research Group, Institute for Infection and Immunity, St George's, University of London, UK, St George's, University of London, UK; ${ }^{2}$ Department of Paediatrics, Imperial College London, UK; ${ }^{3}$ Department of Paediatrics, Northwick Park Hospital, London, UK; ${ }^{4}$ Department of Paediatrics and Child Public Health, University College Hospital, Ibadan, Nigeria

\subsection{6/archdischild-2018-rcpch.318}

Introduction Transverse myelitis (TM) is a neurological disorder causing acute cord injury as a result of acute inflammation, and it is often associated with infectious or autoimmune disease. $20 \%$ of all cases of TM occur in children.

Since 2015, an outbreak of Zika Virus infection (ZiKV) has been reported in over 30 countries. Emerging evidence suggest $\mathrm{ZikV}$ causes a spectrum of neurologic diseases. However, its association with TM, especially in children, is not well described.

Methods We undertook a systematic review of the English literature published from 1947 to August 2017 to evaluate the risk factors, distribution, pathogenesis, clinical presentation, management and outcomes of TM following Zika virus infection. Data sources included MEDLINE, EMBASE, Cochrane library, and references within identified articles. We also searched the papers using the ISI web of knowledge, to identify relevant articles and conference proceedings.

Results We identified 102 potential studies, of which 9 were duplicates and 89 were excluded on the basis of title and abstracts. Of the 4 eligible studies [5-8], there were 6275 with suspected $\mathrm{ZiKV}$ in all age groups. 695 cases were confirmed ZiKV either by RT-PCR in plasma, CSF and urine, ELIZA or MRI while excluding other aetiologies. There were $11(1.6 \%)$ cases of TM. Among 3 studies reporting clinical characteristics and outcome, the mean age was 23 years (Range $15-43)$ and $63 \%(n=5 / 8)$ of cases were male. $40 \%$ $(n=4 / 10)$ required admission to the ITU, with no reported case fatality.

Conclusions and clinical implications

Complications from ZiKV, although uncommon, may be severe. With international spread, clinicians need to be aware that $\mathrm{ZiKV}$ may be associated with TM. As only $10 \%$ of cases were children, standardising the collection and reporting for individual cases across regions and countries would further allow meaningful analysis of the data collected, enabling monitoring of trends over time.

\section{G329(P) RISK OF SEIZURE FOLLOWING ZIKA VIRUS INFECTION IN CHILDREN}

${ }^{1} \mathrm{~L}$ Pay, ${ }^{2} \mathrm{O}$ Oligbu, ${ }^{1} \mathrm{M}$ Fallaha, ${ }^{3} \mathrm{~L}$ Ahmed, ${ }^{4} \mathrm{P}$ Saroey, ${ }^{5} \mathrm{G}$ Oligbu. ${ }^{1}$ Department of Paediatrics, Imperial College London, UK; ${ }^{2}$ Accident and Emergency, Queen Elizabeth Hospital, Woolwich, London, UK; ${ }^{3}$ Accident and Emergency, Chiaring Cross Hospital, London, UKi ${ }^{4}$ Paediatric Infectious Disease, St George's Hospital, London, UK; ${ }^{5}$ Paediatric Infectious Disease Research Group, St George's University of London, UK

\subsection{6/archdischild-2018-rcpch.319}

Introduction Since 2015, an outbreak of Zika Virus infection $(\mathrm{ZiKV})$ has been reported in over 30 countries. Emerging evidence suggest ZikV causes a spectrum of neurologic diseases both directly and by secondary autoimmunity. In pregnancy, $\mathrm{ZiKV}$ is a well-known cause of congenital brain abnormalities, including microcephally, and has been linked with GuillainBarre syndrome. However, its association with seizures in children is not well described.

Methods We undertook a systematic review of the English literature published from 1947 to August 2017 to evaluate the risk factors, epidemiology, clinical presentation, management and outcomes of seizure following Zika virus infection in children less than 18 years. Data sources included MEDLINE, EMBASE, Cochrane library, and references within identified articles. We also searched the papers using the ISI web of knowledge, to identify relevant articles and conference proceedings.

Results We identified 140 potential studies, of which 8 were duplicate and 122 were excluded on the basis of title and abstracts. A further 2 additional studies did not meet eligibility criteria. Of the 8 eligible studies [3-10], there were 215 with confirmed $\mathrm{ZiKV}$ in children less than 18 years by either RTPCR in plasma, CSF and urine, EEG and MRI while excluding other etiologies. 20\% $(n=44)$ cases of had seizure. Among 5 studies reporting clinical characteristics and outcome, the mean age was 5 months (Range 1-8 months) and $75 \%$ $(n=12 / 16)$ of cases were female. All had abnormal neuroimaging $(n=44)$ and responded to anti-convulsant therapy, with no reported mortality.

Conclusions and clinical implications Complications from $\mathrm{ZiKV}$, although uncommon, may be severe. With international spread, clinicians need to be aware that ZiKV may be associated with seizures in children. Therefore, standardising the collection and reporting for individual cases across regions and countries would further allow meaningful analysis of the data collected, enabling monitoring of trends over time.

\section{Association of Paediatric Emergency Medicine}

\section{G330 THE PREVIEW TRIAL - A PROSPECTIVE, RANDOMISED, DOUBLE-BLIND, PLACEBO CONTROLLED TRIAL ASSESSING ORAL PREDNISOLONE IN PRE-SCHOOL AGED CHILDREN WITH VIRAL ASSOCIATED WHEEZE}

${ }^{1} \mathrm{SJ}$ Foster, ${ }^{2} \mathrm{MN}$ Cooper, ${ }^{1} \mathrm{~S}$ Oosterhof, ${ }^{1,3} \mathrm{ML}$ Borland. 'Paediatric Emergency Department, Princess Margaret Hospital for Children, Perth, Western Australia, Australia; ${ }^{2}$ Telethon Kids Institute, The University of Western Australia, Perth, Australia; ${ }^{3}$ Divisions of Paediatrics and Emergency Medicine, School of Medicine, University of Western Australia, Perth, Australia

\subsection{6/archdischild-2018-rcpch.320}

Background Pre-school aged children experience frequent episodes of wheeze that follow a different clinical course to 